\title{
ERRATUM
}

\section{Vicia faba-Lygus rugulipennis Interactions: Induced Plant Volatiles and Sex Pheromone Enhancement}

\author{
Francesca Frati - Keith Chamberlain - Michael Birkett - Samuel Dufour • \\ Patrick Mayon • Christine Woodcock • Lester Wadhams • John Pickett • \\ Gianandrea Salerno $\cdot$ Eric Conti $\cdot$ Ferdinando Bin
}

Published online: 24 April 2009

(C) Springer Science + Business Media, LLC 2009

\section{Erratum to: J Chem Ecol (2009) 35:201-208 \\ DOI 10.1007/s10886-008-9572-6}

The presentation of Table 1 in the original version of this article unfortunately contained a mistake. The corrected table is given below. Springer regrets the error.

Table 1 Mean amount \pm SE of volatiles collected from healthy Vicia faba plants, plants damaged by Lygus rugulipennis males that had been removed, plants damaged by females that had been removed, plants damaged by males still present, plants damaged by females still present

\begin{tabular}{|c|c|c|c|c|c|c|c|}
\hline \multirow[t]{3}{*}{ Treatment } & \multirow[t]{3}{*}{$N$} & \multicolumn{6}{|c|}{ Compounds $(\mathrm{ng} / \mathrm{h})^{\mathrm{a}}$} \\
\hline & & \multicolumn{2}{|l|}{ Alcohols } & \multirow[t]{2}{*}{ Alkaloids Indole } & \multicolumn{3}{|l|}{ Esters } \\
\hline & & (Z)-3-Hexenol & 1-Hexanol & & (Z)-3-hexenyl acetate & Hexyl acetate & Methyl salicylate \\
\hline Healthy & 7 & $194.86 \pm 40.87 \mathrm{a}$ & $2.69 \pm 0.70 \mathrm{a}$ & $1.82 \pm 0.44 \mathrm{a}$ & $55.28 \pm 16.42 \mathrm{a}$ & $0.36 \pm 0.20 \mathrm{~b}$ & $0.44 \pm 0.04 \mathrm{c}$ \\
\hline Damaged by $\widehat{\jmath}$ & 7 & $224.07 \pm 65.14 \mathrm{a}$ & $2.37 \pm 0.57 \mathrm{a}$ & $2.29 \pm 0.58 \mathrm{a}$ & $47.13 \pm 10.04 \mathrm{a}$ & $0.09 \pm 0.06 \mathrm{~b}$ & $2.00 \pm 0.54 b c$ \\
\hline Damaged by $q$ & 7 & $196.80 \pm 48.34 \mathrm{a}$ & $1.96 \pm 0.41 \mathrm{a}$ & $2.27 \pm 0.48 \mathrm{a}$ & $52.11 \pm 9.94 \mathrm{a}$ & $0.20 \pm 0.15 \mathrm{ab}$ & $2.86 \pm 0.65 \mathrm{ab}$ \\
\hline Damaged $+\hat{\sigma}$ & 7 & $193.29 \pm 34.98 \mathrm{a}$ & $2.04 \pm 0.33 \mathrm{a}$ & $3.51 \pm 1.33 \mathrm{a}$ & $41.47 \pm 9.90 \mathrm{a}$ & $0.42 \pm 0.24 \mathrm{ab}$ & $2.40 \pm 0.58 b$ \\
\hline Damaged $+q$ & 7 & $94.90 \pm 34.21 \mathrm{a}$ & $1.82 \pm 0.50 \mathrm{a}$ & $2.95 \pm 1.13 \mathrm{a}$ & $81.55 \pm 46.48 \mathrm{a}$ & $1.41 \pm 0.41 \mathrm{a}$ & $8.90 \pm 2.98 \mathrm{a}$ \\
\hline
\end{tabular}

Total duration of volatile collection was $24 \mathrm{~h}$, starting at $\sim 11.00-12.00 \mathrm{am}$

${ }^{\mathrm{a}}$ The mean amount of each compound (ng/h) was estimated by comparison of peak area with that of an internal standard (dodecane, $\left.100 \mathrm{ng}\right)$. Numbers in column followed by the same letter indicate means that are not significantly different $(P>0.05$, ANOVA).

$N$ number of replicates, TMTT $(E, E)$-4,8,12-trimethyl-1,3,7,11-tridecatetraene

The online version of the original article can be found at http://dx.doi. org/10.1007/s10886-008-9572-6.

F. Frati $(\bowtie) \cdot$ G. Salerno $\cdot$ E. Conti $\cdot$ F. Bin

Dipartimento di Scienze Agrarie e Ambientali-Entomologia,

University of Perugia,

Borgo XX Giugno 74,

06121 Perugia, Italy

e-mail: francescafrati@tiscali.it

K. Chamberlain - M. Birkett - S. Dufour $\cdot$ P. Mayon •

C. Woodcock $\cdot$ L. Wadhams $\cdot$ J. Pickett

Biological Chemistry Division, Rothamsted Research,

Harpenden, Hertfordshire AL5 2JQ, UK 